\title{
Dynamical Regularity for Action Analysis
}

Vinay Venkataraman ${ }^{1}$

http://www.public.asu.edu/ vvenka18/

loannis Vlachos ${ }^{2}$

ivlachos@latech.edu

Pavan Turaga ${ }^{1}$

http://www.public.asu.edu/ pturaga/
${ }^{1}$ School of Arts, Media + Engineering,

School of Electrical, Computer and Energy Engineering,

Arizona State University,

Arizona, USA

${ }^{2}$ Center for Biomedical Engineering and Rehabilitation Science, Louisiana Tech University,

Louisiana, USA
An activity can be seen as a resultant of coordinated movement of body joints and their respective interdependencies to achieve a goal-directed task. This idea is further supported by Johansson's demonstrations that visual perception of the entire human body motion can be represented by a few bright spots which holistically describe the motion of important joints. Traditional dynamical modeling approaches usually operate on the level of individual joints of the human body, lacking any information about the interdependencies between joints. We propose a novel approach for dynamical modeling by extending conventional ideas to quantify the interdependencies between body joints. Towards this end, we propose a new approach - approximate entropy-based feature representation to model the dynamics in human movement by quantifying dynamical regularity. In this paper, we utilize the algorithmic framework of [3] for estimating approximate entropy from time series data and extend it to model the dynamics in human activities for applications such as temporal segmentation and fine-grained quality assessment of actions.

Approximate entropy is a statistical tool proposed by Pincus [3, 4] for quantification of regularity of time series data and system complexity, based on the log-likelihood of repetitions of patterns of length $m$ being close within a defined tolerance window that will exhibit similar characteristics as patterns of length $(m+1)$ [2,3]. It assigns a non-negative number to time series data, with lower values for predictable (ordered) signals and higher values for signals with increased irregularity (or randomness). It is defined using three parameters: embedding dimension $(m)$, radius $(r)$, and time delay $(\tau)$. Here, $m$ represents the length of pattern (also called as embedding vector) in the data which is checked for repeatability, $\tau$ is selected so that the components of the embedding vector are sufficiently independent, and $r$ is used for the estimation of local probabilities. Given $N$ data samples $\left\{x_{1}, x_{2}, \ldots, x_{N}\right\}$, we can define embedding vector $\mathbf{x}(i)$ as,

$$
\mathbf{x}(i)=\left[x_{i}, x_{i+\tau}, \ldots, x_{i+(m-1) \tau}\right]^{T} ; \quad \text { for } \quad 1 \leq i \leq N-(m-1) \tau .
$$

The frequency of repeatable patterns of the embedding vector within a tolerance $r$ is given by $\mathbf{C}_{i}^{m}(r)$ as

$$
\mathbf{C}_{i}^{m}(r)=\frac{1}{N-(m-1) \tau} \sum_{<j>} \Theta(r-d(\mathbf{x}(i), \mathbf{x}(j))) .
$$

Approximate Entropy is given by

$$
\operatorname{ApEn}(m, r, \tau)=\Phi^{m}(r)-\Phi^{m+1}(r) .
$$

where:

$$
\Phi^{m}(r)=\frac{1}{N-(m-1) \tau} \sum_{i=1}^{N-(m-1) \tau} \ln \mathbf{C}_{i}^{m}(r) .
$$

$\mathbf{C}_{i}^{m}(r)$ represents the frequency of repeatable patterns in the embedding vector $\mathbf{x}(i), \Theta(a)$ is the Heaviside step function, and $\Phi^{m}(r)$ represents the conditional frequency estimates.

Multivariate Cross Approximate Entropy (XAPEN): Recent theoretical and empirical findings have demonstrated that multivariate embedding of time series data by simple concatenation of individual univariate embedding vectors achieves good state space reconstruction as evaluated by the shape and dynamics distortion measures. In this work, we propose to use the multivariate embedding procedure as described by Cao et al. [1] per body joint and estimate the approximate entropy feature representation. In addition, natural human movement involves multiple body joints interacting with each other to together accomplish a particular action task. Hence, it would be beneficial to utilize the cross-coupling information between these joint trajectories. We use cross approximate

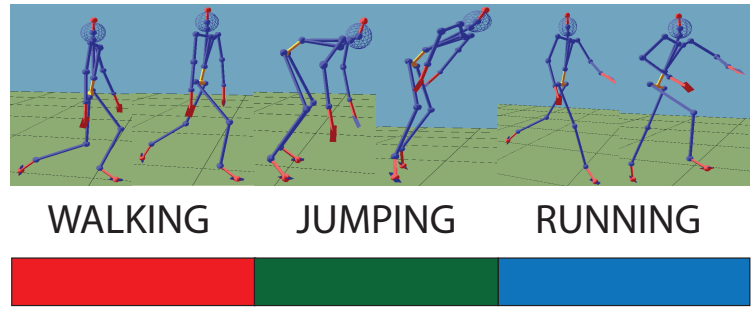

(a) Temporal segmentation of actions using motion capture data.
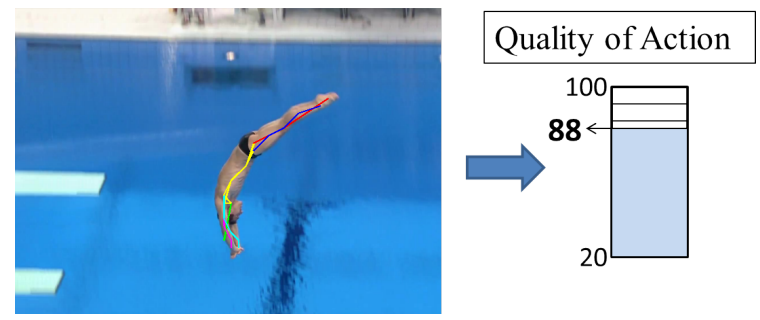

(b) Quality assessment of diving actions using videos.

Figure 1: A visual representation of our applications of interest in this work. In (a), our aim is to achieve temporal segmentation of actions from continuous untrimmed motion capture data in an unsupervised manner. In (b), we use a supervised learning framework to assess the quality of diving actions from videos.

entropy to quantify the cross-coupling information between body joints, with frequency of repeatable patterns within the embedding vectors $\mathbf{x}_{1}(i)$ and $\mathbf{x}_{2}(i)$ generated from two time series data given by $\mathbf{C}_{i}^{m}(r)(v \| u)$ as

$$
\mathbf{C}_{i}^{m}(r)(v \| u)=\frac{1}{N-(m-1) \tau} \sum_{<j>} \Theta\left(r-d\left(\mathbf{x}_{1}(i), \mathbf{x}_{2}(j)\right)\right) .
$$

We estimate the cross approximate entropy feature across all pairs of body joints after performing multivariate embedding using data available from each body joint. We then concatenate ApEn and XApEn values to form our final approximate entropy-based feature vector to model actions. The principle herein is to quantify regularity (frequency of typical patterns) in the dynamical space computed from trajectories of action data. Our experimental evaluation on theoretical models and two publicly available databases show that the proposed features can achieve state-ofthe-art results on applications such as temporal segmentation and quality assessment of actions.

Acknowledgments: This work was supported in part by National Science Foundation (NSF) CAREER grant 1452163 and NSF 1320267.

[1] Liangyue Cao, Alistair Mees, and Kevin Judd. Dynamics from multivariate time series. Physica D: Nonlinear Phenomena, 121(1):75-88, 1998.

[2] Steve Pincus and Burton H Singer. Randomness and degrees of irregularity. Proceedings of the National Academy of Sciences, 93(5): 2083-2088, 1996.

[3] Steven M Pincus. Approximate entropy as a measure of system complexity. Proceedings of the National Academy of Sciences, 88(6): 2297-2301, 1991.

[4] Steven M Pincus. Irregularity and asynchrony in biologic network signals. Methods in enzymology, 321:149-182, 2000. 